H.-X. YI

KODAI MATH. J.

18 (1995), 300-314

\title{
UNICITY THEOREMS FOR MEROMORPHIC FUNCTIONS THAT SHARE THREE VALUES
}

\author{
HONG-XUN YI
}

This paper studies the problem of the uniqueness of meromorphic functions that share three values. The results in this paper improve some theorems given by $\mathrm{H}$. Ueda, Shou-Zhen Ye and Hong-Xun Yi. Examples are provided to show that our results are sharp.

\section{Introduction and main results}

Let $f$ and $g$ be two nonconstant meromorphic functions in the complex plane. If $f$ and $g$ have the same $a$-points with the same multiplicities, we say $f$ and $g$ share the value a $C M$. (see [1]). It is assumed that the reader is familiar with the basic notations and fundamental results of Nevanlinna's theory of meromorphic functions, as found in [2]. It will be convenient to let $E$ denote any set of positive real numbers of finite linear measure, not necessarily the same at each occurrence. The notation $S(r, f)$ denotes any quantity satisfying

$$
S(r, f)=o(T(r, f)) \quad(r \rightarrow \infty, r \notin E) .
$$

H. Ueda proved the following theorem.

THEOREM A (see [3]). Let $f$ and $g$ be two distinct nonconstant entire functions such that $f$ and $g$ share 0, 1 CM., and let $a$ be a finite complex number, and $a \neq 0,1$. If $a$ is lacunary for $f$, then $1-a$ is lacunary for $g$, and

$$
(f-a)(g+a-1) \equiv a(1-a) .
$$

In [4] the present author proved the following result which is an improvement of the above result.

THEOREM B. Let $f$ and $g$ be two distinct nonconstant entire functions such that $f$ and $g$ share 0,1 CM., and let $a$ be a finite complex number, and $a \neq 0,1$.

1991 Mathematics Subject Classification 30D35, 30D30, 30D20.

* Project Supported by the National Natural Science Foundation of PRC.

Received October 4, 1993; revised June 6, 1994. 
If $\delta(a, f)>1 / 3$, then $a$ and $1-a$ are Picard exceptional values of $f$ and $g$ respectively, and

$$
(f-a)(g+a-1) \equiv a(1-a) .
$$

Recently Shou-Zhen Ye extended the above theorems to meromorphic functions, and proved the following theorems.

THEOREM C (see [5]). Let $f$ and $g$ be two distinct nonconstant meromorphic functions such that $f$ and $g$ share $0,1, \infty C M$., and let $a$ be a finite complex number, and $a \neq 0,1$. If

$$
\delta(a, f)+\delta(\infty, f)>\frac{4}{3},
$$

then $a$ and $1-a$ are Picard values of $f$ and $g$ respectively, and also $\infty$ is so, and

$$
(f-a)(g+a-1) \equiv a(1-a) .
$$

THEOREM D (see [5]). Let $f$ and $g$ be two distinct nonconstant meromorphic functions such that $f$ and $g$ share $0,1, \infty C M$., and let $a_{1}, a_{2}, \cdots, a_{p}$ be $p(\geqq 1)$ distinct finite complex numbers, and $a_{j} \neq 0,1(j=1,2, \cdots, p)$. If

$$
\sum_{\jmath=1}^{p} \delta\left(a_{\jmath}, f\right)+\delta(\infty, f)>\frac{2(p+1)}{p+2}
$$

then there exists one and only one $a_{k}$ in $a_{1}, a_{2}, \cdots, a_{p}$ such that $a_{k}$ and $1-a_{k}$ are Picard values of $f$ and $g$ respectively, and also $\infty$ is so, and

$$
\left(f-a_{k}\right)\left(g+a_{k}-1\right) \equiv a_{k}\left(1-a_{k}\right) .
$$

In this paper we improve the above theorems and obtain the following results.

THEOREM 1. Let $f$ and $g$ be two distinct nonconstant meromorphuc functions such that $f$ and $g$ share $0,1, \infty C M$., and let $a$ be a finite complex number, and $a \neq 0,1$. If

$$
N\left(r, \frac{1}{f-a}\right) \neq T(r, f)+S(r, f)
$$

and

$$
N(r, f) \neq T(r, f)+S(r, f),
$$

then $a$ and $1-a$ are Picard values of $f$ and $g$ respectively, and also $\infty$ is so, and

$$
(f-a)(g+a-1) \equiv a(1-a) .
$$

By Theorem 1 we immediately obtain the following result which is an improvement of Theorems A, B, C and D. 
THEOREM 2. Let $f$ and $g$ be two distint nonconstant meromorphac functions such that $f$ and $g$ share $0,1, \infty C M$., and let $a$ be a finite complex number, and $a \neq 0,1$. If $\delta(a, f)>0$ and $\delta(\infty, f)>0$, then $a$ and $1-a$ are Picard values of $f$ and $g$ respectively, and also $\infty$ is so, and

$$
(f-a)(g+a-1) \equiv a(1-a) .
$$

Example 1. Let $f(z)=\left(e^{2 z}+1\right) /\left(e^{z}+1\right), g(z)=\left(e^{-2 z}+1\right) /\left(e^{-z}+1\right), \quad a=2 . \quad$ It is easy to see that $f$ and $g$ share $0,1, \infty C M$., and

$$
N(r, f) \neq T(r, f)+S(r, f)
$$

and $\delta(\infty, f)=1 / 2>0$. Noting

$$
f(z)-a=\frac{e^{2 z}-2 e^{z}-1}{e^{z}+1},
$$

we have

$$
N\left(r, \frac{1}{f-a}\right)=T(r, f)+S(r, f)
$$

and $\delta(a, f)=0 . \quad(f-a)(g+a-1) \neq \equiv a(1-a)$ is evident.

Example 2. Let $f(z)=2 /\left(1+e^{z}\right), g(z)=2 /\left(1+e^{-z}\right), a=2$. It is easy to see that $f$ and $g$ share $0,1, \infty C M$., and

$$
N(r, f)=T(r, f)+S(r, f)
$$

and $\delta(\infty, f)=0$. Noting

$$
f(z)-a=-\frac{2 e^{z}}{1+e^{z}}
$$

we have

$$
N\left(r, \frac{1}{f-a}\right) \neq T(r, f)+S(r, f)
$$

and $\delta(a, f)=1>0 . \quad(f-a)(g+a-1) \not \equiv a(1-a)$ is evident.

Example 3. Let $f(z)=1 /\left(e^{z}+1\right), g(z)=1 /\left(e^{-z}+1\right), a=2$. It is easy to see that $f$ and $g$ share $0,1, \infty C M$., and

$$
N(r, f)=T(r, f)+S(r, f)
$$

and $\delta(\infty, f)=0$. Noting

$$
f(z)-a=-\frac{2 e^{z}+1}{e^{z}+1},
$$

we have

$$
N\left(r, \frac{1}{f-a}\right)=T(r, f)+S(r, f)
$$


and $\delta(a, f)=0 . \quad(f-a)(g+a-1) \not \equiv a(1-a)$ is evident.

The above examples show that Theorem 1 and Theorem 2 are sharp.

\section{Some lemmas}

The following lemmas will be needed in the proof of our theorems.

LEMMA 1. Let $f$ and $g$ be two distinct nonconstant meromorphic functions such that $f$ and $g$ share $0,1, \infty C M$., then

$$
f=\frac{e^{q}-1}{e^{p}-1}, \quad g=\frac{e^{-q}-1}{e^{-p}-1},
$$

where $p$ and $q$ are entire functions such that $e^{p} \not \equiv 1, e^{q} \neq 1$ and $e^{q-p} \not \equiv 1$, and

$$
T(r, g)+T\left(r, e^{p}\right)+T\left(r, e^{q}\right)=O(T(r, f)) \quad(r \notin E) .
$$

Proof. By assumption we have with two entire functions $\alpha$ and $\beta$,

$$
f=e^{\alpha} \cdot g \text { and } f-1=e^{\beta} \cdot(g-1) .
$$

Since $f \not \equiv g$, then $e^{\alpha} \not \equiv 1, e^{\beta} \not \equiv 1$ and $e^{\beta-\alpha} \not \equiv 1$. Setting $p=\beta-\alpha$ and $q=\beta$, we have $e^{p} \not \equiv 1, e^{q} \not \equiv 1$ and $e^{q-p} \not \equiv 1$. Thus from this we get

$$
f=\frac{e^{q}-1}{e^{p}-1} \text { and } g=\frac{e^{-q}-1}{e^{-p}-1} .
$$

By the second fundamental theorem, we have

$$
\begin{aligned}
T(r, g) & <N\left(r, \frac{1}{g}\right)+N\left(r, \frac{1}{g-1}\right)+N(r, g)+S(r, g) \\
& =N\left(r, \frac{1}{f}\right)+N\left(r, \frac{1}{f-1}\right)+N(r, f)+S(r, g) \\
& <3 T(r, f)+S(r, g) .
\end{aligned}
$$

Thus from this we get

$$
\begin{gathered}
T\left(r, e^{q}\right)=T\left(r, e^{\beta}\right) \leqq \\
<(r, f-1)+T(r, g-1)+O(1) \\
<4 T(r, f)+S(r, g), \\
T\left(r, e^{q-p}\right)=T\left(r, e^{\alpha}\right) \leqq T(r, f)+T(r, g)+O(1) \\
<4 T(r, f)+S(r, g), \\
T\left(r, e^{p}\right)=T\left(r, e^{q-(q-p)}\right) \leqq T\left(r, e^{q}\right)+T\left(r, e^{q-p}\right)+O(1) \\
<8 T(r, f)+S(r, g) .
\end{gathered}
$$

Hence 


$$
T(r, g)+T\left(r, e^{p}\right)+T\left(r, e^{q}\right)=O(T(r, f)) \quad(r \notin E) .
$$

This completes the proof of Lemma 1 .

LEMma 2. Let $f$ and $g$ be two nonconstant meromorphic functions, and let $c_{1}, c_{2}$ and $c_{3}$ be three nonzero constants. If

then

$$
c_{1} f+c_{2} g \equiv c_{3},
$$

$$
T(r, f)<\bar{N}\left(r, \frac{1}{f}\right)+\bar{N}\left(r, \frac{1}{g}\right)+\bar{N}(r, f)+S(r, f) .
$$

Proof. By the second fundamental theorem, we have

$$
\begin{aligned}
T(r, f) & <\bar{N}\left(r, \frac{1}{f}\right)+\bar{N}\left(r,\left(f-\frac{c_{3}}{c_{1}}\right)^{-1}\right)+\bar{N}(r, f)+S(r, f) \\
& =\bar{N}\left(r, \frac{1}{f}\right)+\bar{N}\left(r, \frac{1}{g}\right)+\bar{N}(r, f)+S(r, f),
\end{aligned}
$$

which proves Lemma 2.

LEMMA 3 (see [6, Lemma 4]). Let $p(z)$ be a nonconstant entire function, then

$$
T\left(r, p^{\prime}\right)=o\left(T\left(r, e^{p}\right)\right) \quad(r \notin E) .
$$

In order to state Lemma 4 , we introduce the following notations.

Let $f$ be a meromorphic function. We denote by $n_{1}(r, f)$ the number of simple poles of $f$ in $|z| \leqq r . \quad N_{1}(r, f)$ is defined in terms of $n_{1}(r, f)$ in the usual way. We further define

$$
N_{2}(r, f)=N(r, f)-N_{1}(r, f) .
$$

In the same way we can define $N_{2}(r, 1 / f)$ and $N_{2}(r, 1 /(f-1))$.

LEMMA 4. Let $f$ and $g$ be two distinct nonconstant meromorphic functions such that $f$ and $g$ share $0,1, \infty C M$., then

$$
N_{2}(r, f)+N_{2}\left(r, \frac{1}{f}\right)+N_{2}\left(r, \frac{1}{f-1}\right)=S(r, f) .
$$

Proof. By Lemma 1 we have

$$
f=\frac{e^{q}-1}{e^{p}-1},
$$

where $p$ and $q$ are entire functions such that $e^{p} \not \equiv 1, e^{q} \neq 1$ and $e^{q-p} \not \equiv 1$, and

$$
T(r, g)+T\left(r, e^{p}\right)+T\left(r, e^{q}\right)=O(T(r, f)) \quad(r \notin E) .
$$

If $e^{q} \equiv c$, where $c(\neq 0,1)$ is a constant, then we have $N(r, 1 / f)=0$. If $e^{q}$ 
is not a constant, let $\left\{z_{n}\right\}$ be all the roots of $f=0$ with multiplicity $\geqq 2$, then $\left\{z_{n}\right\}$ are the roots of $\left(e^{q}-1\right)^{\prime}=q^{\prime} e^{q}=0$. Thus

$$
N_{2}\left(r, \frac{1}{f}\right) \leqq 2 N\left(r, \frac{1}{q^{\prime}}\right) \leqq 2 T\left(r, q^{\prime}\right)+O(1) .
$$

Again by Lemma 3, we deduce

$$
N_{2}\left(r, \frac{1}{f}\right)=S(r, j)
$$

If $e^{p} \equiv c$, where $c(\neq 0,1)$ is a constant, then we have $N(r, f)=0$. If $e^{p}$ is not a constant, let $\left\{z_{n}\right\}$ be all the roots of $1 / f=0$ with multiplicity $\geqq 2$, then $\left\{z_{n}\right\}$ are the roots of $\left(e^{p}-1\right)^{\prime}=p^{\prime} e^{p}=0$. Thus

$$
N_{2}(r, f) \leqq 2 N\left(r, \frac{1}{p^{\prime}}\right) \leqq 2 T\left(r, p^{\prime}\right)+O(1) .
$$

Again by Lemma 3, we have

Note that

$$
N_{2}(r, f)=S(r, f) .
$$

$$
f(z)-1=\frac{e^{p}\left(e^{q-p}-1\right)}{e^{p}-1} .
$$

If $e^{q-p} \equiv c$, where $c(\neq 0,1)$ is a constant, then $N(r, 1 /(f-1))=0$. If $e^{q-p}$ is not a constant, let $\left\{z_{n}\right\}$ be all the roots of $f-1=0$ with multiplicity $\geqq 2$, then $\left\{z_{n}\right\}$ are the roots of $\left(e^{q-p}-1\right)^{\prime}=\left(q^{\prime}-p^{\prime}\right) e^{q-p}=0$. Thus

$$
N_{2}\left(r, \frac{1}{f-1}\right) \leqq 2 N\left(r, \frac{1}{q^{\prime}-p^{\prime}}\right) \leqq 2 T\left(r, q^{\prime}\right)+2 T\left(r, p^{\prime}\right)+O(1) .
$$

Again by Lemma 3, we have

$$
N_{2}\left(r, \frac{1}{f-1}\right)=S(r, f) .
$$

From the above three equalities, we obtain

$$
N_{2}(r, f)+N_{2}\left(r, \frac{1}{f}\right)+N_{2}\left(r, \frac{1}{f-1}\right)=S(r, f),
$$

which proves Lemma 4.

\section{Proof of Theorem 1}

By the assumption, from Lemma 1 we have

$$
f=\frac{e^{q}-1}{e^{p}-1}, \quad g=\frac{e^{-q}-1}{e^{-p}-1},
$$


where $p$ and $q$ are entire functions such that $e^{p} \not \equiv 1, e^{q} \not \equiv 1$ and $e^{q-p} \not \equiv 1$, and

$$
T(r, g)+T\left(r, e^{p}\right)+T\left(r, e^{q}\right)=O(T(r, f)) \quad(r \notin E) .
$$

We discuss the following four cases.

a) Suppose that $e^{p} \equiv c(\neq 0,1)$.

By (1) we have

$$
f=\frac{e^{q}-1}{c-1}
$$

and

$$
f-a=\frac{e^{q}-1-a(c-1)}{c-1} .
$$

If $-1-a(c-1) \neq 0$, from $(4)$,

$$
N\left(r, \frac{1}{f-a}\right)=T(r, f)+S(r, f),
$$

which contradicts the assumption of Theorem 1 . Then $-1-a(c-1)=0$ and $c=$ $(a-1) / a$. Again by (1), we obtain

and

$$
f=a-a e^{q}
$$

$$
g=(1-a)-(1-a) e^{-q} .
$$

Thus a and $1-a$ are Picard values of $f$ and $g$ respectively, and also $\infty$ is so, and

$$
(f-a)(g+a-1) \equiv a(1-a) .
$$

b) Suppose that $e^{q} \equiv c(\neq 0,1)$.

By (1) we have

$$
f=\frac{c-1}{e^{p}-1} .
$$

Thus

$$
N(r, f)=T(r, f)+S(r, f),
$$

which contradicts the assumption of Theorem 1.

c) Suppose that $e^{q-p} \equiv c(\neq 0,1)$.

By (1) we have

$$
f=\frac{c e^{p}-1}{e^{p}-1}=c+\frac{c-1}{e^{p}-1} .
$$

Thus

$$
N(r, f)=T(r, f)+S(r, f),
$$

which is again a contradiction. 
d) Suppose that none of $e^{p}, e^{q}$ and $e^{q-p}$ are constants.

It is clear that $p^{\prime} \not \equiv 0, q^{\prime} \neq 0$ and $p^{\prime} \not \equiv q^{\prime}$. By Lemma 1 and Lemma 3 , we have

$$
T\left(r, p^{\prime}\right)+T\left(r, q^{\prime}\right)=S(r, f) .
$$

Set

$$
h=\frac{q^{\prime}}{p^{\prime}} .
$$

From (5) and (6) we obtain $h \not \equiv 0,1$, and

If

$$
T(r, h)=S(r, f) .
$$

$$
q^{\prime}(h-1)-h^{\prime} \equiv 0 \text {, }
$$

by integration, we have

$$
h-1=c_{1} e^{q},
$$

where $c_{1}$ is a constant, and $c_{1} \neq 0$. From (6) and (7), we obtain

$$
\frac{q^{\prime}}{c_{1} e^{q}+1}=p^{\prime}
$$

Again by integration, we get

$$
c_{1}+e^{-q}=c_{2} e^{-p},
$$

where $c_{2}$ is a constant, and $c_{2} \neq 0$. Thus

$$
c_{2} e^{-p}-e^{-q}=c_{1} .
$$

By Lemma 2, we obtain

$$
T\left(r, e^{-p}\right)=S\left(r, e^{-p}\right),
$$

which is impossible. Hence

$$
q^{\prime}(h-1)-h^{\prime} \not \equiv 0
$$

From (1), we have

$$
f-h=\frac{e^{q}-h e^{p}+h-1}{e^{p}-1} .
$$

Set

$$
F=(f-h)\left(e^{p}-1\right)=e^{q}-h e^{p}+h-1,
$$

then

$$
\frac{F^{\prime}}{F}-q^{\prime}=\frac{\left(e^{q}-h e^{p}+h-1\right)^{\prime}-q^{\prime}\left(e^{q}-h e^{p}+h-1\right)}{(f-h)\left(e^{p}-1\right)}
$$




$$
=\frac{q^{\prime}(h-1)-h^{\prime}}{f-h}
$$

and hence

$$
\frac{1}{f-h}=\frac{\left(F^{\prime} / F\right)-q^{\prime}}{q^{\prime}(h-1)-h^{\prime}} .
$$

From (9) we get

$$
m\left(r, \frac{1}{f-h}\right) \leqq m\left(r, \frac{F^{\prime}}{F}\right)+S(r, f)=S(r, f)
$$

and

$$
N_{2}\left(r, \frac{1}{f-h}\right)=S(r, f) .
$$

Again from (1), we have

$$
\frac{f-g}{g-1}=e^{q}-1
$$

and

$$
\frac{g^{\prime}}{g}=\frac{q^{\prime} e^{p}-p^{\prime} e^{q}+\left(p^{\prime}-q^{\prime}\right)}{\left(e^{q}-1\right)\left(e^{p}-1\right)} .
$$

Thus

$$
\frac{g^{\prime}(f-g)}{g(g-1)}=\frac{q^{\prime} e^{p}-p^{\prime} e^{q}+\left(p^{\prime}-q^{\prime}\right)}{e^{p}-1} .
$$

From (6) and (8), we obtain

$$
-p^{\prime}(f-h)=\frac{q^{\prime} e^{p}-p^{\prime} e^{q}+\left(p^{\prime}-q^{\prime}\right)}{e^{p}-1} .
$$

By (12) and (13), we get

$$
-p^{\prime}(f-h)=\frac{g^{\prime}(f-g)}{g(g-1)} .
$$

Again by Lemma 4 and (11), we have

$$
N\left(r, \frac{1}{f-h}\right)=N\left(r, \frac{1}{g^{\prime}}\right)+N_{0}(r)+S(r, f),
$$

where $N_{0}(r)$ denotes the counting function of the zeros of $f-g$ that are not zeros of $g$ and $g-1$. From (10) and (15), we obtain

$$
\begin{aligned}
T(r, f) & =T(r, f-h)+S(r, f) \\
& =m\left(r, \frac{1}{f-h}\right)+N\left(r, \frac{1}{f-h}\right)+S(r, f)
\end{aligned}
$$


MEROMORPHIC FUNCTIONS

309

$$
=N\left(r, \frac{1}{g^{\prime}}\right)+N_{0}(r)+S(r, f) \text {. }
$$

Thus

$$
T(r, f)-N\left(r, \frac{1}{g^{\prime}}\right)=N_{0}(r)+S(r, f) .
$$

In the same manner as above, we have

$$
T(r, g)-N\left(r, \frac{1}{f^{\prime}}\right)=N_{0}(r)+S(r, f) .
$$

By the second fundamental theorem, and using (16), we obtain

$$
\begin{aligned}
T(r, f)+T(r, g) \leqq & T(r, f)+N\left(r, \frac{1}{g}\right)+N\left(r, \frac{1}{g-1}\right)+N(r, g) \\
& -N\left(r, \frac{1}{g^{\prime}}\right)+S(r, f) \\
= & N\left(r, \frac{1}{g}\right)+N\left(r, \frac{1}{g-1}\right)+N(r, g)+N_{0}(r)+S(r, f) \\
\leqq & N\left(, r \frac{1}{f-g}\right)+N(r, g)+S(r, f) \\
\leqq & T(r, f-g)+N(r, g)+S(r, f) \\
\leqq & m(r, f)+m(r, g)+N(r, f-g)+N(r, g)+S(r, f) \\
\leqq & m(r, f)+m(r, g)+N(r, f)+N(r, g)+S(r, f) \\
= & T(r, f)+T(r, g)+S(r, f) .
\end{aligned}
$$

Thus

$$
T(r, f)+T(r, g)=N\left(r, \frac{1}{g}\right)+N\left(r, \frac{1}{g-1}\right)+N(r, g)+N_{0}(r)+S(r, f) .
$$

Again by the second fundamental theorem, and using (17) and (18), we have

$$
\begin{aligned}
2 T(r, f) \leqq & N\left(r, \frac{1}{f}\right)+N\left(r, \frac{1}{f-1}\right)+N\left(r, \frac{1}{f-a}\right) \\
& +N(r, f)-N\left(r, \frac{1}{f^{\prime}}\right)+S(r, f) \\
\leqq & N\left(r, \frac{1}{g}\right)+N\left(r, \frac{1}{g-1}\right)+N\left(r, \frac{1}{f-a}\right) \\
& +N(r, g)+N_{0}(r)-T(r, g)+S(r, f) \\
= & T(r, f)+N\left(r, \frac{1}{f-a}\right)+S(r, f)
\end{aligned}
$$


Thus

$$
\leqq 2 T(r, f)+S(r, f) \text {. }
$$

$$
N\left(r, \frac{1}{f-a}\right)=T(r, f)+S(r, f),
$$

which contradicts the assumption of Theorem 1.

This completes the proof of Theorem 1.

\section{Applications of Theorem 1}

For any set $S$ and any meromorphic function $f$ let

$$
E_{f}(S)=\bigcup_{a \in S}\{z \mid f(z)-a=0\},
$$

where each zero of $f-a$ with multiplicity $m$ is repeated $m$ times in $E_{f}(S)$ (see [9]).

Recently the present author corrected a result of Gross and Yang [11] and proved the following theorems.

Theorem E (see [10]). Let $S_{1}=\left\{a_{1}, a_{2}\right\}$ and $S_{2}=\left\{b_{1}, b_{2}\right\}$ be two pairs of distinct elements with $a_{1}+a_{2}=b_{1}+b_{2}$ but $a_{1} a_{2} \neq b_{1} b_{2}$, and let $S_{3}=\{\infty\}$. Suppose that $f$ and $g$ are two nonconstant meromorphic functions satisfying $E_{f}\left(S_{\jmath}\right)=E_{g}\left(S_{\jmath}\right)$ for $j=1,2,3$. Then $N(r, f)=S(r, f)$ and

$$
T(r, f)=T(r, g)+S(r, f) .
$$

THEOREM $\mathrm{F}$ (see [10]). If, in addition to the assumptions of Theorem E, $\delta(c / 2, f)>1 / 5$, where $c=a_{1}+a_{2}$, then $f$ and $g$ must satisfy exactly one of the following relations:

(i) $f \equiv g$,

(ii) $f+g \equiv a_{1}+a_{2}$, $\left.b_{2}\right)^{2}=0$.

(iii) $(f-c / 2)(g-c / 2) \equiv \pm\left(\left(a_{1}-a_{2}\right) / 2\right)^{2}$. This occurs only for $\left(a_{1}-a_{2}\right)^{2}+\left(b_{1}-\right.$

TheOrem G (see [10]). If, in addition to the assumptions of Theorem E,

$$
N\left(r, \frac{1}{f-b_{1}}\right)+N\left(r, \frac{1}{f-b_{2}}\right)=2 T(r, f)+S(r, f)
$$

and $\delta(c / 2, f)>0$, where $c=a_{1}+a_{2}$, then the conclusions of Theorem $F$ hold.

Applying Theorem 1, we immediately obtain the following result which is an improvement of Theorem $\mathrm{F}$ and Theorem $\mathrm{G}$.

THEOREM 3. If, in addition to the assumptions of Theorem $E, \delta(c / 2, f)>0$, where $c=a_{1}+a_{2}$, then the conclusions of Theorem $F$ hold. 
Proof. By Theorem E we have

$$
N(r, f) \neq T(r, f)+S(r, f) .
$$

Again by $\delta(c / 2, f)>0$, we also have

$$
N\left(r, \frac{1}{f-c / 2}\right) \neq T(r, f)+S(r, f) .
$$

Let

$$
F=\frac{(f-c / 2)^{2}-\left(\left(a_{1}-a_{2}\right) / 2\right)^{2}}{\left(\left(b_{1}-b_{2}\right) / 2\right)^{2}-\left(\left(a_{1}-a_{2}\right) / 2\right)^{2}}, \quad G=\frac{(g-c / 2)^{2}-\left(\left(a_{1}-a_{2}\right) / 2\right)^{2}}{\left(\left(b_{1}-b_{2}\right) / 2\right)^{2}-\left(\left(a_{1}-a_{2}\right) / 2\right)^{2}} .
$$

If $F \equiv G$, it is obvious that $f \equiv g$ or $f+g \equiv a_{1}+a_{2}$. Next, assume that $F \not \equiv G$.

By $E_{f}\left(S_{j}\right)=E_{g}\left(S_{j}\right)(j=1,2,3)$, we know that $F$ and $G$ share $0,1, \infty C M$. From (19) and (20), we have

and

$$
N(r, F) \neq T(r, F)+S(r, F)
$$

$$
N\left(r, \frac{1}{F-a}\right) \neq T(r, F)+S(r, F)
$$

where

$$
a=\frac{-\left(\left(a_{1}-a_{2}\right) / 2\right)^{2}}{\left(\left(b_{1}-b_{2}\right) / 2\right)^{2}-\left(\left(a_{1}-a_{2}\right) / 2\right)^{2}} \neq 0,1 .
$$

By Theorem 1, it follows that $a$ is a Picard value of $F$, and hence $c / 2$ is a Picard value of $f$. Thus $\delta(c / 2, f)=1>1 / 5$. Again by Theorem $\mathrm{F}$, we obtain

$$
(f-c / 2)(g-c / 2) \equiv \pm\left(\left(a_{1}-a_{2}\right) / 2\right)^{2},
$$

this occurs only for $\left(a_{1}-a_{2}\right)^{2}+\left(b_{1}-b_{2}\right)^{2}=0$.

This also completes the proof of Theorem 3 .

Example 4. Let $f(z)=1-4 e^{z}, g(z)=1-e^{-z}, a_{1}=-1, a_{2}=1, b_{1}=-\sqrt{3} i, b_{2}=$ $\sqrt{3} i, S_{1}=\left\{a_{1}, a_{2}\right\}, S_{2}=\left\{b_{1}, b_{2}\right\}, S_{3}=\{\infty\}$. It is easy to verify that

$$
\frac{\left(f-a_{1}\right)\left(f-a_{2}\right)}{\left(g-a_{1}\right)\left(g-a_{2}\right)}=-8 e^{3 z}, \quad \frac{\left(f-b_{1}\right)\left(f-b_{2}\right)}{\left(g-b_{1}\right)\left(g-b_{2}\right)}=4 e^{2 z},
$$

which show $E_{f}\left(S_{j}\right)=E_{g}\left(S_{\jmath}\right)$ for $j=1,2,3$. Thus, $f$ and $g$ satisfy the conditions of Theorem E. Noting that $c=a_{1}+a_{2}=0$ and $f(z)-c / 2=1-4 e^{z}$, we have $\delta(c / 2, f)=0 . \quad f \not \equiv g, \quad f+g \not \equiv a_{1}+a_{2}$ and $(f-c / 2)(g-c / 2) \not \equiv \pm\left(\left(a_{1}-a_{2}\right) / 2\right)^{2}$ are evident. This shows that Theorem 3 is sharp.

\section{Improvement of Theorem 1}

Proceeding as in the proof of Theorem 1, we can prove the following result, which is an improvement of Theorem 1 . 
THEOREM 4. Let $f$ and $g$ be two distinct nonconstant meromorphic functions such that $f$ and $g$ share $0,1, \infty C M$., and let $a$ be a finite complex number, and $a \neq 0,1$. If

$$
N\left(r, \frac{1}{f-a}\right) \neq T(r, f)+S(r, f),
$$

then $a$ is a Picard exceptional value of $f$, and $f$ and $g$ must satisfy exactly one of the following relations:

(i) $(f-a)(g+a-1) \equiv a(1-a)$. This occurs only for $\infty$ be a Picard exceptional value of $f$. In this case, $1-a$ and $\infty$ are Picard exceptional values of $g$.

(ii) $f+(a-1) g \equiv a$. This occurs only for 0 be a Picard exceptional value of $f$. In this case, $a /(a-1)$ and 0 are Picard exceptional values of $g$.

(iii) $f \equiv a g$. This occurs only for 1 be a Picard exceptional value of $f$. In this case, $1 / a$ and 1 are Picard exceptional values of $g$.

Proof. Proceeding as in the proof of Theorem 1, we can obtain (1).

We discuss the following four cases.

a) Suppose that $e^{p} \equiv c(\neq 0,1)$.

Proceeding as in the proof of Theorem 1, we can obtain the relation (i), and $a$ and $\infty$ are Picard exceptional values of $f, 1-a$ and $\infty$ are Picard exceptional values of $g$.

b) Suppose that $e^{q} \equiv c(\neq 0,1)$.

By (1) we have

$$
f=\frac{c-1}{e^{p}-1}
$$

and

$$
f-a=\frac{(c-1+a)-a e^{p}}{e^{p}-1} .
$$

If $c-1+a \neq 0$, from (21),

$$
N\left(r, \frac{1}{f-a}\right)=T(r, f)+S(r, f),
$$

which contradicts the assumption of Theorem 4 . Then $c-1+a=0$ and $c=1-a$. Again by (1), we obtain

$$
f=-\frac{a}{e^{p}-1}
$$

and

$$
g=\frac{a e^{p}}{(a-1)\left(e^{p}-1\right)}
$$

Thus, we get the relation (ii), and $a$ and 0 are Picard exceptional values of $f$, $a /(a-1)$ and 0 are Picard exceptional values of $g$. 
c) Suppose that $e^{q-p} \equiv c(\neq 0,1)$.

By (1) we have

$$
f=\frac{c e^{p}-1}{e^{p}-1}
$$

and

$$
f-a=\frac{(c-a) e^{p}-(1-a)}{e^{p}-1} .
$$

If $c-a \neq 0$, from (22),

$$
N\left(r, \frac{1}{f-a}\right)=T(r, f)+S(r, f),
$$

which contradicts the assumption of Theorem 4. Then $c=a$. Again by (1), we obtain

$$
f=\frac{a e^{p}-1}{e^{p}-1}
$$

and

$$
g=\frac{a e^{p}-1}{a\left(e^{p}-1\right)} .
$$

Thus, we get the relation (iii), and $a$ and 1 are Picard exceptional values of $f, 1 / a$ and 1 are Picard exceptional values of $g$.

d) Suppose that none of $e^{p}, e^{q}$ and $e^{q-p}$ are constants.

Proceeding as in the proof of Theorem 1, we can arrive at a contradiction.

This completes the proof of Theorem 4.

By Theorem 4 we immediately obtain the following corollary.

COROLLARY. Let $f$ and $g$ be two nonconstant meromorphic functions such that $f$ and $g$ share $0,1, \infty C M$., and let $a$ be a finite complex number, and $a \neq 0,1$. If

$$
N\left(r, \frac{1}{f-a}\right) \neq T(r, f)+S(r, f),
$$

and none of $0,1, \infty$ are Picard exceptional values of $f$, then $f \equiv g$.

Acknowledgement. I am grateful to the referee for valuable comments.

\section{REFERENCES}

[1] G.G. Gundersen, Meromorphic functions that share three or four values, J. London Math. Soc., 20 (1979), 457-466.

[2] W. K. Hayman, Meromorphic Functions, Clarendon Press, Oxford, 1964.

[3] H. UEDA, Unicity theorems for meromorphic or entire functions, Kodai Math. J., 3 (1980), 457-471. 
[4] Hong-XUn YI, Meromorphic functions that share three values, Chinese Ann. Math., 9A (1988), 434-439.

[5] Shou-Zhen Ye, Uniqueness of meromorphic functions that share three values, Kodai Math. J., 15 (1992), 236-243.

[6] Hong-Xun YI, Meromorphic functions that share two or three values, Koda1 Math. J., 13 (1990), 363-372.

[7] Hong-XUN YI, Unicity theorems for meromorphic functions, J. Shandong Univ., 23 (1988), 15-22.

[8] G. BRosch, Eindeutigkeltssätze für meromorphc Funktıonen, Thesıs, Technical University of Aachen, 1989.

[9] F. Gross, On the distribution of values of meromorphic functions, Trans. Amer. Math. Soc., 131 (1968), 199-214.

[10] Hong-XUN YI, On a result of Gross and Yang, Tôhoku Math. J., 42 (1990), 419-428.

[11] F. Gross AND C.C. YANG, Moromorphic functions covering certain finite sets at the same points, Illinois J. Math., 26 (1982), 432-441.

Department of Mathematics

SHANDONG UNIVERSITY

JiNAN, SHANDONG, 250100

People's Republic of China 